\title{
Modeling of nanomaterials: recent applications and new tools development G Goldbeck-Wood
}

\author{
Address: Accelrys, 334 Cambridge Science Park, Cambridge, CB4 OWN, UK \\ from 3rd German Conference on Chemoinformatics \\ Goslar, Germany. II-I3 November 2007 \\ Published: 26 March 2008 \\ Chemistry Central Journal 2008, 2(Suppl I):S7 doi:I0.II86/I752-I53X-2-SI-S7
}

This abstract is available from: http://www.journal.chemistrycentral.com/content/2/SI/S7

(c) 2008 Goldbeck-Wood

Modeling and simulation of nanomaterials and devices require realistic description of the system across various length and time scales. In this regard, a multiscale modeling approach of the system starting from quantum mechanical modeling to describe the electronic structure and optical properties, atomistic molecular dynamics (MD) calculations to describe diffusion processes, and meso scale simulation to describe the morphology of soft materials takes prominence. In this paper, applications in the materials sector are presented including the design of polymer nanotube composites, resins with particular property profiles sensor materials and fuel cell catalysts.

Following from those studies further requirements for modeling tools emerge, and the talk will discuss recent and future developments. These include an integrated atomistic and mesoscale modeling framework for polymer materials, a toolset for reactive systems (covering areas such as catalysis and adhesives), including linear scaling DFT, QM/MM and reactive forcefield methods, and combinatorial approaches enabled by data pipelining and mining.

Finally, the frameworks in which these development take place will be described including the Nanotechnology Consortium. This consortium was initiated by Accelrys in 2004 with the aim to deliver state-of-the-art nanotechnology software tools. It has grown into a global collaboration of currently 29 members, and has delivered a range of new software tools. Plans for its second phase from 2007 to 2010 will be discussed. 